\title{
BougieCap Dilation in Eosinophilic Esophagitis for Stricture Treatment under Visual and Haptic Control
}

\author{
Vincent Zimmer ${ }^{1,2}$ \\ ${ }^{1}$ Department of Medicine, Marienhausklinik St. Josef Kohlhof, \\ Neunkirchen, Germany \\ ${ }^{2}$ Department of Medicine II, Saarland University Medical Center, \\ Saarland University, Homburg, Germany
}

J Digest Endosc:2020;11:223-224

A 51-year-old male with a recently established diagnosis of stricturing eosinophilic esophagitis (EoE) under antiinflammatory treatment with orodispersible budesonide 1-mg bid presented for esophageal stricture treatment due to persistent dysphagia. The recent ("subacute") esophagogastroduodenoscopy (EGD) indicated discrete EoE stigmata, such as edema with reduced vascular pattern, discrete linear furrows, and rings ( - Fig. 1A), as well as a hardly passable stricture of an estimated 2-cm axial length in the distal esophagus ( $\mathbf{- F i g . ~ 1 B )}$ ), summing up to an endoscopic
Address for correspondence Vincent Zimmer, MD, Department of Medicine, Marienhausklinik St. Josef Kohlhof, Klinikweg 1-5, 66539 Neunkirchen, Germany (e-mail: vincent.zimmer@gmx.de).

reference score (EREFS) of 5 points. The patient underwent esophageal dilation without fluoroscopy using a 12- and 14-mm BougieCap (Ovesco Endoscopy, Tübingen, Germany) as an innovative novel endoscopy accessory for stricture treatment under visual and haptic control. ${ }^{1}$ During slow and judicious increases in forward forces within the stricture with marked circumferential mucosal whitening reflective of tissue traction, mucosal lacerations and tears at 7 and 11 o'clock ( - Fig. 1C) and later on at 1 o'clock became visible. ( - Fig. 1D) The further clinical course was uncomplicated,
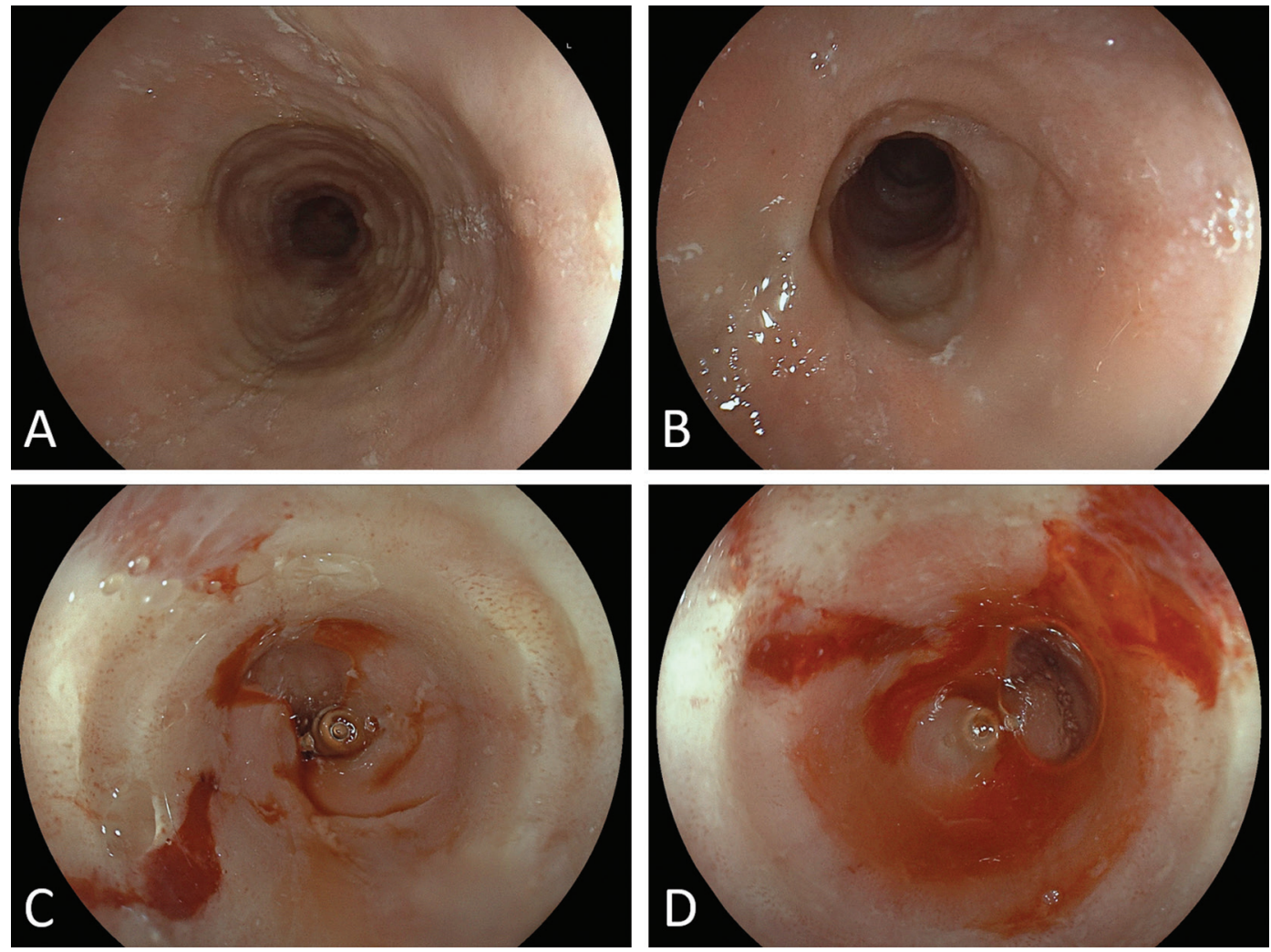

Fig. 1 (A) Endoscopic image of the midesophagus illustrating signs of eosinophilic esophagitis (EoE) with reduced vascular pattern, discrete linear furrows, and rings, as well as (B) an estimated 2-cm stricture in the distal esophagus (EREFS score 5). (C) BougieCap dilation procedure with circumferential mucosal whitening reflective of tissue traction and minor mucosal lacerations at 7 and 11 o'clock. (D) Later stage of the procedure highlighting a larger tear at 1 o'clock.

DOI https://doi.org/ $10.1055 / \mathrm{s}-0040-1716586$ ISSN 0976-5042. 


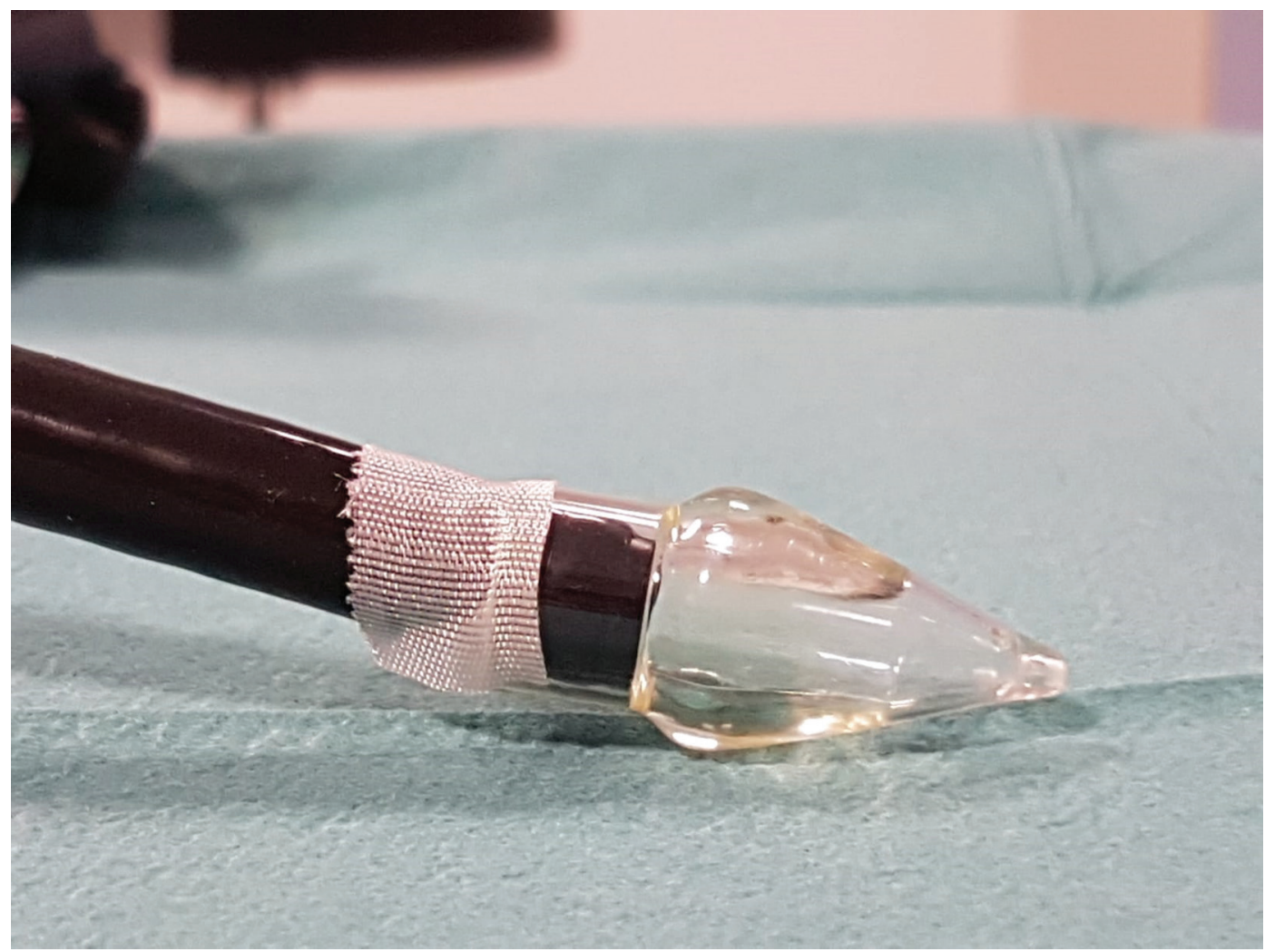

Fig. 2 The BougieCap device slipped on and being taped to the end of an upper endoscope.

and the patient underwent another dilation session 6 weeks apart up to $16 \mathrm{~mm}$ with durable clinical and endoscopic response ( - Video 1; available in the online version).

The BougieCap, as a radiolucent single-use, domeshaped transparent cap of different available sizes from 7 to $16 \mathrm{~mm}$, is attached to the scope tip. (-Fig. 2) Bougienage is by gently advancing the endoscopy through the stricture under ancillary rotational movements under both endoscopic and haptic control. Whether or not a guidewire used for BougieCap dilation is at the discretion of the operator. In our endoscopy practice, guidewire assistance is implemented for high-grade and/or proximal strictures. In addition, we suggest using a guidewire in the first procedures of individual operators during the learning curve. Preliminary experience in real-world use and a recent systematic study have established its overall safety in endoscopy practice with "the lost cap" being a signature, albeit mostly innocuous complication. ${ }^{2}$ Whether or not its use translates into improved safety in esophageal dilation in the high-risk population of EoE patients, notorious for oftentimes impressive mucosal tears, and postdilation chest pain awaits further research. ${ }^{3}$

\section{Financial Disclosure}

The author discloses no financial relationships relevant to this publication.

\section{Video 1}

BougieCap dilation in eosinophilic esophagitis for stricture treatment under visual and haptic control. Online content including video sequences viewable at: https:// www.thieme-connect.com/products/ejournals/html/ doi/10.1055/s-0040-1716586.

\section{Conflict of Interest}

The author states no conflict of interest.

\section{References}

1 Ramrakhiani $\mathrm{H}$, Triadafilopoulos G. Negotiating dire straits with a BougieCap. Dig Dis Sci 2020

2 Walter B, Schmidbaur S, Rahman I, Albers D, Schumacher B, Meining A. The BougieCap - a new method for endoscopic treatment of complex benign esophageal stenosis: results from a multicenter study. Endoscopy 2019;51(9):866-870

3 Zimmer V. BougieCap esophageal passage: a novel potential tool for calibration of lumen diameter in eosinophilic esophagitis? Gastrointest Endosc 2018;88(3):568-569 